\title{
Plasmonic Electricity: Fluorophore-Induced Plasmonic Current
}

\author{
Joshua Moskowitz and Chris D. Geddes* \\ Institute of Fluorescence and Department of Chemistry and Biochemistry, \\ University of Maryland Baltimore County, Baltimore, MD 21202, USA \\ *All Correspondence: Geddes@umbc.edu
}

\section{Supporting Information}
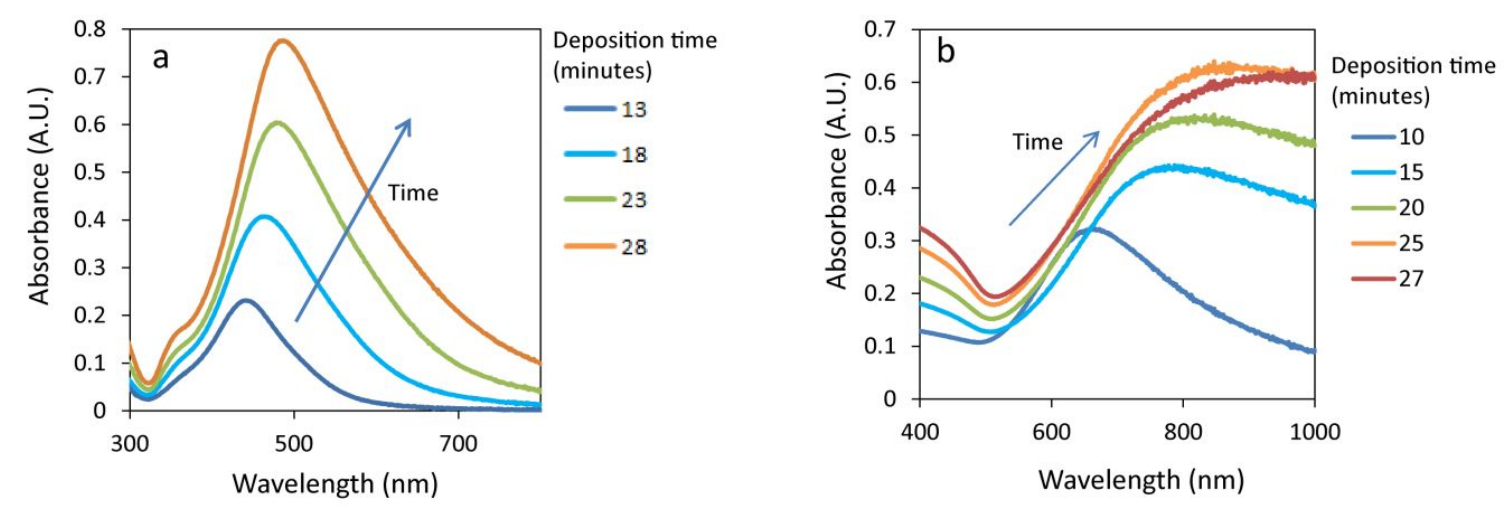

Figure S1: Absorption spectra for silver (a) and gold (b) over a range of thermal vapor deposition times. All films were prepared at a deposition rate of $0.1 \AA /$ second.

\section{Model For Capacitance of a Metal Nanoparticle Island Film}

Capacitance of an individual metal nanoparticle in the film is estimated with the concentric sphere model. ${ }^{24,26-29}$

$$
\mathrm{C}=4 \pi_{\varepsilon 0 \varepsilon} \mathrm{r}_{0}\left(\mathrm{r}_{0}+s\right) / s
$$

where $\varepsilon_{0}$ is the vacuum permittivity, $\varepsilon$ is the relative permittivity of the medium surrounding the particle, $r_{0}$ is the particle radius, and $s$ is the distance between two neighbouring particles.

$\mathrm{C}$ is then substituted into the grid model for capacitance of a large grid containing many identical capacitors. ${ }^{33}$

$$
\frac{\mathrm{C}_{\mathrm{m}, \mathrm{n}}}{\mathrm{C}}=\frac{\pi}{2 \ln \left(\mathrm{m}^{2}+\mathrm{n}^{2}\right)+4 \ln \pi+0.22} \quad \text { (Eq. 4) }
$$

where $C$ is the capacitance of a single nanoparticle, $C_{m, n}$ is the capacitance between two nanoparticles on opposite sides of the nanoparticle film, and $(m, n)$ are 
coordinates on the grid of nanoparticles where an electron enters the grid at $(0,0)$ and exits at $(m, n)$.

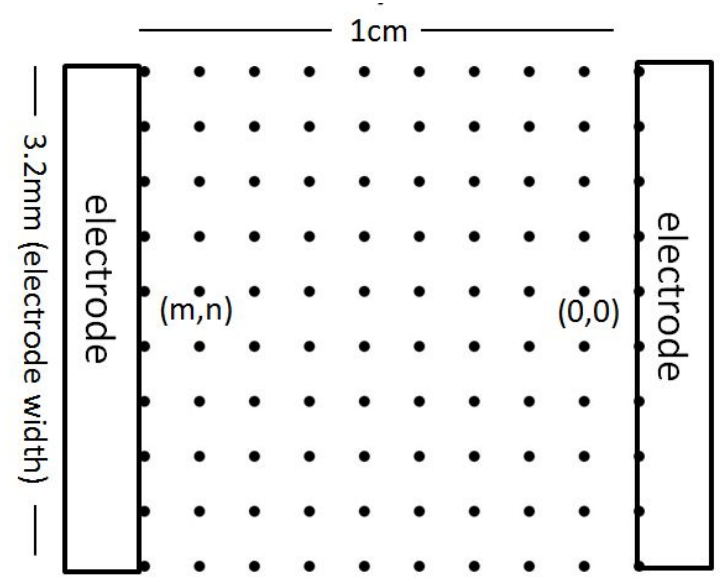

Figure S2: Grid model for capacitance of a metal nanoparticle island film. Each point on the grid represents a metal nanoparticle with a known capacitance (C).

$\mathrm{C}_{\mathrm{m}, \mathrm{n}}$ is then multiplied by the number of nanoparticles directly adjacent to the electrode surface, giving the theoretical film capacitance $\left(C_{\text {film }}\right)$. Following substitution and rearrangement, the model takes the form,

$$
\mathrm{C}_{\mathrm{film}}=\frac{\pi\left(4 \pi_{\varepsilon_{0}} \mathrm{r}_{0}\left(\mathrm{r}_{0}+\mathrm{s}\right) / \mathrm{s}\right)}{2 \ln \left(\mathrm{m}^{2}+\mathrm{n}^{2}\right)+4 \ln \pi+0.22} \times \frac{\text { electrode width }}{2 \mathrm{r}_{0}+\mathrm{s}} \quad \text { (Eq. 5) }
$$

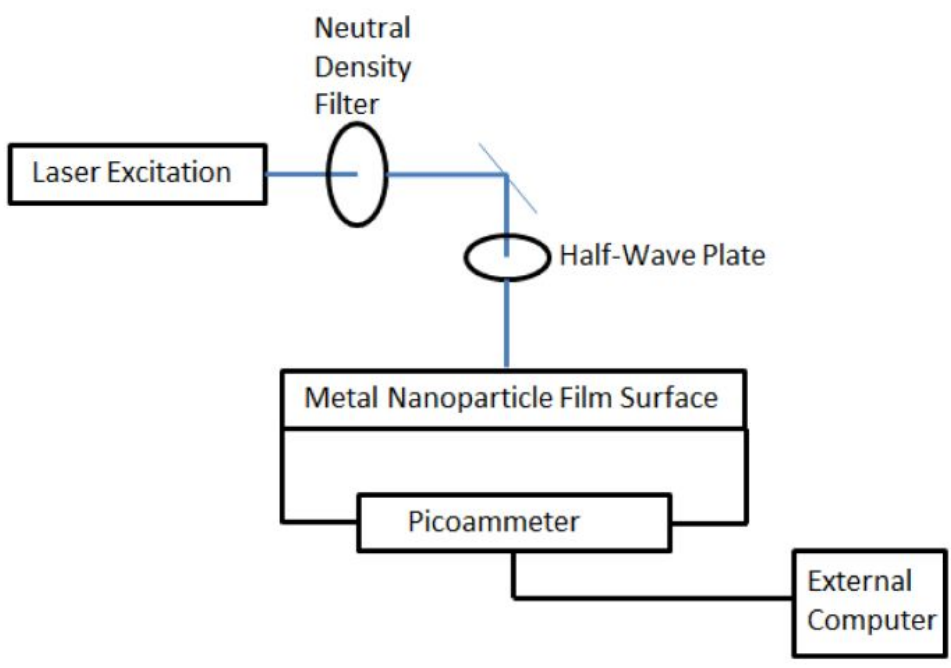

Figure S3: Experimental setup depicting an excitation laser directed incident onto a metal nanoparticle film surface, connected in circuit with a picoammeter. 